\title{
Exploration and Thinking on the Complementarity of Multiple Learning Methods in Community Education
}

\section{Yunshi Fang}

Guangzhou College of Technology and Business, Fang Yunshi, Guangzhou City, Guangdong Province, 510850

Project Source: Youth Project of Foshan Social Science Planning Project in 2020_ Application Research of Model A in Foshan Community Education (Project No. 2020-QN12)

\begin{abstract}
With the gradual improvement of community construction, people gradually shift their focus from infrastructure construction to diversified educational needs, and the promotion of diversified learning community vehicle inspection has become an important model for future community development. This article first discusses the status quo of diversified learning in community education, and explores and ponders the complementarity of diversified learning methods in community education based on what we have learned, in order to further improve the quality and effect of community education.
\end{abstract}

Keywords: Community education; multiple learning methods; complementarity; exploration and thinking

Community education is an important learning method for people to acquire knowledge and skills in the current era. With the continuous deepening and development of community education, it has effectively promoted the extension and expansion of traditional classroom learning to diversified forms, forming the most common and popular variety at present. The method of learning can accurately and effectively meet the various learning needs of citizens. In the process of further research, each learning method has its own unique advantages. How to integrate them to the greatest extent to form an effective complement has become a key issue that education researchers need to consider. Combining many years of educational work experience, the author conducts research and exploration on the complementarity between the diverse learning methods of community education, with the aim of better serving the public and satisfying the increasingly diversified knowledge needs.

\section{An Analysis of the Characteristics of Diversified Learning in Community Education}

Community education was originally developed to make up for the insufficiency of community activities. Therefore, the learning methods shown are diverse and different. After a lot of research and sorting, the author summarizes these learning methods into the following five categories:

First, classroom learning.

Classroom learning is a common form of community education, including organized educational lectures, learning of various interest courses, etc. The main feature is that the content taught is very systematic, and there is strong organizational discipline in the teaching process, one-time education Large numbers, very efficient and practical.

Second, active learning.

Activity learning refers to a way of learning based on community activities. The main common forms are cultural and sports activities, art performances, cultural tourism, and various festival theme activities. This learning method is very flexible and attractive to the people, effectively increasing the people's learning enthusiasm.

Copyright (C) 2020 Yunshi Fang

doi: 10.18282/le.v9i6.1337

This is an open-access article distributed under the terms of the Creative Commons Attribution Non-Commercial License

(http://creativecommons.org/licenses/by-nc/4.0/), which permits unrestricted non-commercial use, distribution, and reproduction in any medium, provided the original work is properly cited. 
Third, team learning.

In community education, team learning is a group learning method, and its learning carriers are very diverse, such as various non-governmental community learning, neighborhood learning sites, public reading clubs, and so on. The people who participate in team learning are organized spontaneously with the same goals, have a desire to learn from self-transcendence, have very high learning autonomy, and play an active role in building a harmonious community.

Fourth, experience learning.

Experiential learning is similar to activity learning, but it is more practical and participatory, and focuses on providing a good learning environment for learners. It is a lifelong learning concept with learners as the center of teaching, which can effectively promote the exchange of community people. Interaction, closer relations between people, making community life more harmonious.

Fifth, digital learning.

Digital learning refers to a way of learning through digital technology, including common online education, mobile learning, and distance learning. The characteristic of this learning method is that it is not restricted by time and space, has very good openness and interaction, can arrange the learning agenda more flexibly, and effectively improve the fragmented time utilization of the people.

\section{Exploration and Thinking on the Complementarity of Multiple Learning Methods in Community Education}

Different learning methods have their own advantages and disadvantages. A single learning method can hardly meet the diverse learning needs of the community. Therefore, a variety of learning methods can be effectively combined to form complementarity to enhance learning effects. The following is a combination of several learning methods to highlight the advantages of complementary learning methods.

\subsection{Effective combination between classroom learning and digital learning}

The advantage of classroom learning is that the learning goals are clear, with a very clear learning plan, and learners can also learn knowledge systematically. However, this traditional learning method is limited by space and time. Many people cannot participate in time due to personal work and other reasons, so it is difficult to conduct complete learning. Although pure digital learning is not limited by time and space, it lacks a corresponding learning environment. It is difficult for learners to stick to the learning process, and they are very susceptible to various surrounding factors, resulting in low learning efficiency. Effectively integrate digital learning with it, and make use of the characteristics of digital learning that are not restricted by time and space to complement classroom learning, which greatly improves the effect of classroom learning and builds a good learning environment for digital learning, which is convenient for teachers and colleges. Effective supervision during the period.

\subsection{Effective combination between active learning and team learning}

Community activity learning is very flexible, and because the content has a certain entertainment tendency, it can attract a large number of people to learn. However, the corresponding organization and management are relatively loose and lack a certain degree of cohesion. The people are easy to give up halfway and it is difficult to persist for a long time. . Therefore, if the self-management concept in team learning is integrated into it on this basis, and effective communication between each student in the activity can be formed, the team cohesion of the learners can be comprehensively improved, making the learning process more continuous and effective. In addition, for the team learning method, active learning can enrich the learning content and it is easier to attract like-minded people to participate. It is a very good way of publicity.

\subsection{Effective combination between experiential learning and classroom digital learning}

Whether it is classroom learning or digital learning, it is basically based on the learning of theoretical knowledge. Because of the lack of certain practical experience, the practical application of theoretical knowledge is very limited. Therefore, the organic combination of classroom learning and experiential learning, and the use of experiential learning can give learners a good and rich practical experience, so as to make up for the lack of practical ability in classroom 
learning. In addition, classroom systemic learning can improve the connotation of experiential learning to a certain extent and make experiential learning more systematic and efficient. The two complement each other and effectively improve the learning quality of the community.

\section{Concluding remarks}

The diversified development of community education is an important content of future community construction. It is a manifestation of community people's pursuit of a higher spiritual civilization and a manifestation of the current life-long learning society. Community education managers should combine the various forms of community learning, make effective combinations according to the characteristics of different forms, and use each other's advantages to complement each other to a certain extent, so as to improve the overall level of community education, create a good community education and cultural environment, and Improve the quality of spiritual life of our people.

\section{References}

1. Song Yifang. Exploration and reflection on the complementarity of multiple learning methods in community education[J]. Vocational Education Forum, 2018, 000(003):101-107.

2. Diao Yuanyuan. Citizen Study Tour: A New Carrier of Community Education Experiential Learning_—Based on the Practice Exploration and Thinking of Chengdu Citizen Study Tour Project[J]. Lifelong Education Research, 2018, 029(001): P.32-37.

3. Shen Guanghui. Exploring the combination of distance education and community education-Relying on the practice and thinking of community education carried out by Radio and Television University [J]. Changzhou Lifelong Education, 2010, 000(001): P.8-11. 